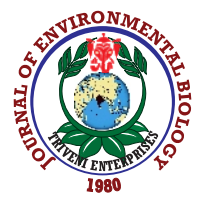

\title{
DNA barcoding analysis of larval fishes in Peninsular Malaysia
}

\author{
I.A. Azmir', Y.B. Esa ${ }^{2,3 *}$, S.M.N. Amin ${ }^{2,4}$, M.Y.I. Salwany ${ }^{2}$ and M.Y.F. Zuraina ${ }^{5}$ \\ 'School of Biology, Faculty of Applied Sciences, Universiti Teknologi MARA (UiTM) Negeri Sembilan, Kampus Kuala Pilah, Pekan Parit Tinggi, \\ 72000 Kuala Pilah, Negeri Sembilan, Malaysia \\ ${ }^{2}$ Department of Aquaculture, Faculty of Agriculture, Universiti Putra Malaysia, 43400 Serdang, Selangor Malaysia \\ ${ }^{3}$ International Institute of Aquaculture and Aquatic Sciences (I-AQUAS), Universiti Putra Malaysia, 71050 port Dickson, Negeri Sembilan, Malaysia \\ ${ }^{4}$ FAO World Fisheries University, Pukyong National University, Busan, 48547, South Korea \\ ${ }^{5}$ Faculty of Applied Sciences, Universiti Teknologi MARA (UiTM), Shah Alam, Selangor, Malaysia \\ *Corresponding Author Email : yuzine@upm.edu.my
}

\begin{abstract}
Aim: To identify fish larvae to species level by DNA barcoding method using the mitochondrial Cytochrome c Oxidase I (COI) gene. The lack of species identification work on fish larvae in Peninsular Malaysia has warrant this study to be conducted.

Methodology: Identification of fish larvae species from mangrove areas of Pendas Johor, Matang Perak, Pekan Pahang and Setiu Terengganu was conducted. Samples were collected from April 2015 to September 2015 using a bongo net, towed at a depth of about $0.5 \mathrm{~m}$ from the surface for 5 min against the tidal flow. From the total of 354 fish larvae collected, a representative of 177 fish larvae was selected and sequenced using COI gene.
\end{abstract}

Results: Results from BLAST and BOLDSYSTEM search showed all sequences had high percentage identity index and similarity $(90 \%$ to $100 \%)$. Fish larvae were identified through phylogenetics analysis showing monophyletic status between query sequences with reference sequences obtained from own collection and GenBank. The Sillago vittata and Sillago sihama sequences was found to be in separate clusters despite their similar genus. A few strong match of specimens from different genus was found with high bootstrap value $(\mathrm{n}>90 \%)$ through Neighbour-Joining (NJ) and Maximumlikelihood (ML) analysis e.g the Paramugil parmatus with Liza melinoptera (NJ $=100 \%, \mathrm{ML}=99 \%$ ) and Pseudogobius oligactis with Eugnathogobius oligactis ( $\mathrm{NJ}=92 \%, \mathrm{ML} 94 \%$ ).

Interpretation: Identification of fish larvae were best conducted with the aid of molecular method, DNA barcoding in particular rather than comparative taxonomical studies alone that able to identify fish larvae sample to genus level at best.

Key words: Cytochrome c Oxidase I (COI) gene, DNA barcoding, Fish larvae, Mangroves, Peninsular Malaysia, Phylogenetic

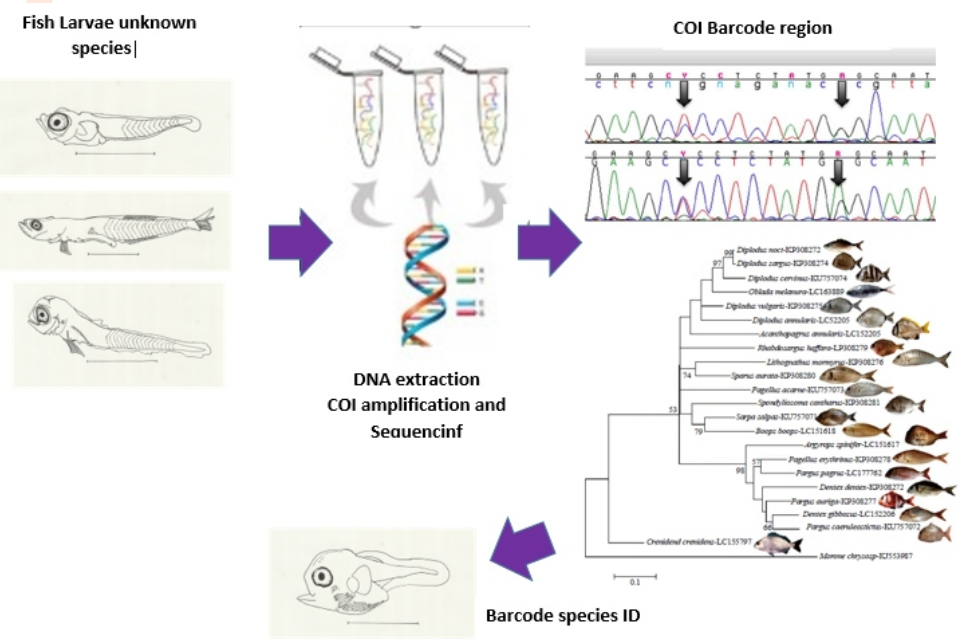

How to cite : Azmir,I.A., Y.B. Esa, S.M.N. Amin, M.Y.I. Salwany and M.Y.F. Zuraina: DNA barcoding analysis of larval fishes in Peninsular Malaysia. J. Environ. Biol., 41, 1295-1308 (2020). 


\section{Introduction}

Malaysia supports a sizeable area of mangrove forest, which covers $505,382 \mathrm{ha}$, and has been automatically ranked as the second largest area covered with mangrove forest among Asian countries (Sandilyan and Kathiresan, 2014). About 99,180 ha of mangrove area is distributed in Peninsular Malaysia (Tan, 2007), and the rest of them are in Sabah and Sarawak. Mangrove is a productive area used by aquatic animals, especially the newly hatched fish eggs and fish larvae. The mangrove ecosystem provides high food sources, low predation rate and stable ocean conditions for growth of its occupants (Frietas and Muelbert, 2004). The fish larvae generally reside in shallow waters as larger predators usually avoid such environments; the magrove (Ara et al., 2011a). However, the vast majority of marine teleost fish have a pelagic larval phase that differs greatly in morphology from the adult. The pelagic stage may last as briefly as 1 week in some damselfish (Pomacentridae) or more than 64 weeks in some porcupine fish (Diodontidae) (Leis and McCormick, 2002). Given this, an established amount of taxonomic key specifically tailored for morphological characteristic identification of fish larvae is required. Otherwise, the identification process will be very challenging considering the rapid development of fish larvae to juvenile stage (short planktonic period) in some fish species, for instance the Damselfish (Wellington and Victor, 1989). As to mediate the process, considerable skills and taxonomic expertise is compulsory (Azmir et al., 2017).

Taxonomic ambiguities have been successfully resolved with the technique called DNA barcoding, which utilizes a short, standardized gene region to identify species (Azmir et al., 2017; Chu et al., 2019). Application of DNA barcoding utilizes the mitochondrial gene instead of nuclear gene as they possess the advantages of having the lack of introns, haploid mode of inheritance, ample selection of primers (Herbert et al., 2003) and a larger number of copies of mitochondrial DNA (mtDNA) present in each cell significantly raises the sensitivity of analysis (Branicki et al., 2003). Thus, the cytochrome c oxidase I (COI) gene was selected for the role of 'universal barcode' considering their relatively high rate of molecular evolution and low in number of insertion or deletion mutations if compared to the ribosomal sequences of 165 (Hebert et al., 2003). According to Ward et al. (2005), COI gene has also proven as a sensitive tool to help discriminate animal identities.

The utilization of mitochondrial DNA, especially cytochrome c oxidase I (COI) gene as a marker in the identification process is fitting as they are present in almost all eukaryotes and have higher evolution rates than nuclear genes, hence confirms it to be the best to discriminate between closely related taxa (Hebert et al., 2003). Through this method, scientists are able to uncover the identity of not only a fully formed organism but also organisms with phenotypically varied or at any stage of development, including egg. In addition, only small amount of
DNA sample retrieved from scale, fin clip, muscle and etc. is enough for amplification and analysis using polymerase chain reaction (PCR) technology (Avise, 1994).

DNA barcoding has made such headway in solving ambiguities of species identification at relatively low cost (Lewis et al., 2016), thus enabling the historically difficult task of linking marine-larval fish to adult becomes more feasible. In addition, DNA barcoding method also enables the identification of microscopic fish larvae down to species level (Shao et al., 2002). Therefore, the aim of this study was to utilize the DNA barcoding method to genetically identify fish larvae samples from selected mangrove areas in Peninsular Malaysia.

\section{Materials and Methods}

Data collection : Fish samples both adults and larvae were collected from mangrove areas in Peninsular Malaysia with seine nets for adults. Fish samples were collected from April 2015 to September 2015. Four mangrove areas were surveyed, namely, Pendas in Johor, Pekan in Pahang, Matang in Perak, and Setiu in Terengganu (Fig. 1). Both Pendas and Matang are located on the west side of Peninsular Malaysia; Pekan and Setiu on the east of Peninsular Malaysia. Sampling was conducted during full moon as moon phases influences the distribution of larval fish species by changing their abundance and composition in the mangrove creeks (Lima et al., 2015). Sampling work was focused during the highest tide by referring to tide tables (National Hydrographic Malaysia, 2015).

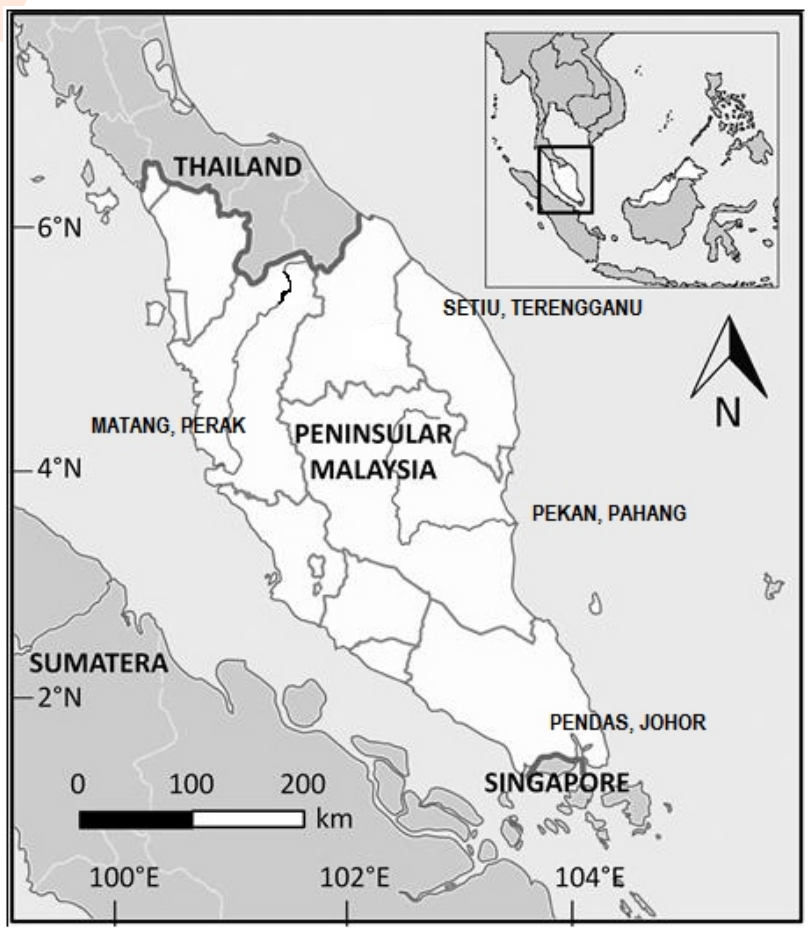

Fig. 1 : Sampling stations of fish larvae from four selected mangrove areas in Peninsular Malaysia (Foon et al., 2015). 
Sampling of larval fish was done with bongo net $(500 \mu \mathrm{m}$ mesh size) towed at $0.5 \mathrm{~m}$ depth from the surface with flow meter attached at the net opening for the rate assessment of water flow. One cycle of towing took $5 \mathrm{~min}$ at the speed of 2 knots. The sampling area took place at the selected mangrove area $\left(50 \mathrm{~m}^{2}\right.$ area surrounding). Sample caught were preserved in $95 \%$ ethanol before it was stored in laboratory. The collection of adult samples was done at the same time and preserved in ice before they were identified to species level by referring to appropriate literature (Atan et al., 2010; Mansor et al., 1998). Details of collection localities were available in Barcode of Life Data System (BOLD) (Ratnasingham and Hebert, 2007) under the project file of 'DNA Barcoding of Fish Larvae from Mangrove areas in Peninsular Malaysia (DNAFL)' and 'DNA Barcoding of Fish from Mangrove areas in Peninsular Malaysia (DBAFM)'.

DNA extraction and purity : DNA extraction of fish larvae and adult fish specimens was done using different kit according to the amount of tissue available. Hence, the extraction method of adult sample was categorized as type $A$, while fish larvae was categorized as type B. Due to ample amount of adult fish tissue sample available, isolation of $20 \mathrm{mg}$ muscle tissue in $1.5 \mathrm{ml}$ microcentrifuge tube was possible. Type A DNA extraction was done using ReliaPrep ${ }^{\mathrm{TM}}$ gDNA Tissue Miniprep System (Promega, USA). The fish larvae were microscopic in size and some of them weighed less than $3 \mathrm{mg}$. Type B of DNA extraction was done with MyTaq ${ }^{\mathrm{TM}}$ Extract-PCR Kit (Bioline, USA). The concentration and purity of DNA were subsequently tested using NanoDrop 1000 V3.8 (Thermo Scientific, USA).

PCR amplification and agarose gel electrophoresis : A fragment of $621 \mathrm{bp}$ to $636 \mathrm{bp}$ of mitochondrial gene was amplified by polymerase chain reaction (PCR), employing primer sets of FishF1 and FishF2 (Ward etal., 2005) at the 5' end of COlgene:

FishF1 (forward): 5'-TCAACCAACCACAAAGACATTGGCAC-3'

FishR1 (reverse): 5'-TAGACTTCT GGGTGGCCAAAGAATCA-3'

FishF2 (forward): 5'- TCGACTAATCATAAAGATATCGGCAC-3'

FishR2 (reverse): 5'-ACTTCAGGGTGACCGAAGAATCAGAA-3'

Amplification reaction was performed in a total volume of $25 \mu \mathrm{l}$, with Promega (Madison, WI) containing $5 X$ Green Fo Taq ${ }^{\circledR}$ Reaction buffer, $25 \mathrm{nM} \mathrm{MgCl}$ solution, deoxynucleotide (dNTP) $40 \mathrm{mM}$, Forward primer 0.1M, Reverse primer 0.1M, Go Taq® DNA polymerase $\left(5 \mu \mu^{-1}\right)$, DNA template $(20 \mathrm{ng})$ and $13.5 \mu$ l of nuclease-free water

The PCR condition was as follows: initial denaturing at $94^{\circ} \mathrm{C}$ for $2 \mathrm{~min}, 35$ cycles of denaturing at $94^{\circ} \mathrm{C}$ for $30 \mathrm{sec}$, annealing at $54^{\circ} \mathrm{C}$ for $50 \mathrm{sec}$, extension at $72^{\circ} \mathrm{C}$ for $40 \mathrm{sec}$ and final extension at $72^{\circ} \mathrm{C}$ for $5 \mathrm{~min}$. PCR mixture was prepared under sterile conditions to ensure contaminant free.
All PCR products were visualized on $1 \%$ agarose gel (Sigma-Aldrich, USA) to check the presence of amplicons of desired size (650bp). Ready to load DNA ladder of $100 \mathrm{bp}$ (Promega, USA) was applied in the well of agarose gel stained with $1 \mu \mathrm{l}$ of GelRed stain (Thermo Fisher Scientific, USA). Electrophoresis condition was set at $80 \mathrm{~V}, 400 \mathrm{~A}, 60 \mathrm{~min}$ in $1 \mathrm{XTBE}$ buffer. Gel products were viewed and documented via Alphalmager HP (Biotechne, USA). Sequencing was done using conventional Sanger sequencing conducted by a private company (First Base Laboratory Sdn. Bhd. Malaysia).

Data analysis : Firstly, the sequences obtained were submitted to BOLD system (http://www.boldsystems.org/) and to BLAST of GenBank (http:// www.ncbi.nlm.nih.gov/) for species identification. Then, the sequencing result of both adult and fish larvae specimens were viewed in Chormas Lite (V 2.1.1) software for trace file viewing and Sequence Scanner (V 2.0) was applied to check on nucleotide base quality. CLUSTALX (V 2.1) (Larkin et al., 2007) was applied for aligning multiple sequences then the trace files were trimmed manually in BioEdit software (V 7.2.5) (Hall, 1999). Haplotype of the sequences were assessed in DsaSP (V 5.10) and then submitted to Barcode of Life Data System (BOLD) (Ratnasingham and Hebert, 2007) for gaining their own barcode status and GenBank to obtain the accession number.

Phylogenetic assessment : The query data set in this study, which was the fish larvae samples, were assessed for their genetic distances among specimens caught. The pairwise genetic distance among intraspecies, intragenus and intrafamily was calculated in BOLD by 'Distance Summary' command. The calculation was based on the Kimura 2-paramater (K2P) (Kimura, 1980) evolutionary model, while the sequences were aligned using MUSCLE algorithm in MEGA 7 software to ensure no gaps nor were stop codons present in the alignment through amino acid translation. A distance analysis using neighbour-joining ( $\mathrm{NJ}$ ) (Saitou and Nei, 1987) analysis was done with a close-neighbourinterchange (CNI) option implemented in MEGA 7 applying the K2P evolutionary model. Phylogenetic confidence was estimated by bootstrapping replicates of 1000 .

Phylogenetic relationship was inferred using three different methods of Bayesian analysis, maximum likelihood (ML) and Neighbour-joining (NJ) (Saitou and Nei, 1987). The bootstrap confidence of 1000 replicates were applied on NJ and ML analyses to evaluate highest likelihood of a tree. Bayesian phylogenetic analysis was done in MrBayes V 3.2.2 (Huelsenbeck and Ronquist, 2001). Markov Chain Monte Carlo (MCMC) process was used to approximate the posterior probabilities of trees which tend to be higher than bootstrap support values calculated in $\mathrm{NJ}$ and $\mathrm{ML}$ tree. The process was set to $4 \times 10^{6}$ generations with tree being sampled every 100 generations. Saturation test was done on all codons using DAMBE V 6.4.29 (Xia, 2013). The trees were rooted using 
sequences of Megaptera novaeangliae (Balaenopteridae) and Hemibagrus nemurus (Bagridae), obtained from GenBank.

\section{Results and Discussion}

DNA barcodes fish larvae species assignments : The discrimination of 38 out of 41 species (93\%) of fish larvae was successfully conducted using DNA barcoding method and further supported with the genetic distance calculation and sequence divergence approach. The results demonstrated the existence of a barcode gap for the species analyzed, thus confirming its effectiveness as a powerful measure for species identification. The presence of barcode gap between lowest interspecific and the highest intraspecific genetic distance indicated correct species identification between samples (Shen et al., 2016). Out of 153 fish larvae sequenced, they corresponded to be the representative of 18 families, 33 genera and 41 species of fish larvae. The number of specimens found per species varied from 1 specimen to 7 specimens per species. Single specimen (singleton) was found in many species including Ambassis sp., Chelonodon patoca, Dendrophysa russelii, Eugnathogobius variegates, Exyrias puntang, Hippichthys cyanospilos, Hippichthys penicillus, Leiognathus ruconius, Monacanthus chinensis, Mugilogobius chulae, Ostorhinchus fasciatus, Paramonacanthus sulcatus, Paramugil parmatus, Photopectoralis bindus. Sillago sihama, Sillago vittata and Upeneus margarethae (Table 1).

The correct discrimination of $95 \%$ of analyzed species (38/41) was done based on the unique haplotypes obtained from 117 barcoded sequences. Out of 117 sequences, 98 haplotypes were assigned with GenBank accession and BOLDSYSTEM index number (Table 2). All amplified fragments of fish larvae specimens inhabiting the mangrove areas of Peninsular Malaysia contained $621 \mathrm{bp}$ and sequences showed good quality, with no evidence of insertions, deletions or stop codon. There were 285 $(45.9 \%)$ conserved sites, $336(54.1 \%)$ variable sites and 288 $(46.4 \%)$ parsimony informative sites found in the partial sequences of successfully amplified mitochondrial $\mathrm{CO} /$ gene. No pseudogene in DNA structure was confirmed through translation of 98 haplotypes for conserved 621 bp fragment containing 206 amino acids.

The within species average K2P distance was $1.00 \%$ compared to $20.19 \%$ for species within genera (Table 3 ). Thus, it was approximately 20 times more divergent than conspecific individuals. This value was an appropriate threshold value according to Hebert et al. (2003) for flagging genetically divergent specimen as definite species. DNA barcoding distinguished species based on nucleotide sequences whenever interspecific variation was greater than the intraspecific (Ajmal Khan et al., 2014).

The mean $\mathrm{K} 2 \mathrm{P}$ distance values were found incongruous for conspecific and congeneric comparisons (1.00\% and $20.19 \%)$ with the ranged recorded in the study of Neotropical freshwater fish where the value was from zero to $8.5 \%$ and from zero to $24.8 \%$ (Pereira et al., 2013); from zero to $3.3 \%$ and from $1.9 \%$ to 28.2\% Yucatan fish (Valdez-Moreno et al., 2010). High number of mean values observed in congeneric species reflected low number of closely related species analyzed. The low number of species analyzed might be a contributing factor to these mean values. Other than that, it was also caused by a few genera represented by only one or more species (Pereira et al., 2013). Thus, an increase in number of species per genus can help in decreasing the mean value of congeneric.

Assignment of fish larvae species were from the public reference records in BOLD Indo-Pacific libraries, GenBank and also based on the adult dataset collected in this study. About $17 \%$ (7 species) of the query matched the own collection of adult datasets, while the remaining query were matched with the reference barcodes libraries (BOLD and GenBank). Successful species assignments were achieved in large number of cases with $98 \%$ and above similarity $(78 \%, 32 / 41)$ with divergent value below $2 \%$. Value of $<2 \%$ for species divergent indicated an extreme distant fish populations among the studied samples (Ward et al., 2008), which at the same time indicated that the populations were part of single species (Hebert et al., 2003) and genetic variability did not increase when the species originated from similar region (Shen et al., 2016).

About $22 \%(9 / 41)$ were found matched to $90-98 \%$ similarity with the reference barcode libraries with $>2 \%$ divergence between species. The exception of having $>2 \%$ divergence occurred in Opisthonema libertate $(2.63 \%)$, Sardinella melanura $(2.79 \%)$, Stolephorus indicus $(2.79 \%)$, Ophiocara porocephala (3.14\%), Periophthalmus sp. (4.51\%), Sicyopterus sp. (6.8\%), Hemigobius hoevenii $2.62 \%)$, Thryssa hamiltonii (3.46\%) and Gerres filamentosus (2.79\%). Nevertheless, all species with $>2 \%$ conspecific divergence were unambiguously identified using $\mathrm{CO}$ barcoding proven by $\mathrm{K} 2 \mathrm{P}$ distance to the nearest neighbours of min $16.01 \%$ (Table 4).

Possibility of species mis-assignment may occur to a morphologically close sister species, especially when there is shortfall in DNA barcode data of species within a given group (Landi et al., 2014). Nevertheless, the K2P distanced to their nearest neighbors were in the range of $16.01 \%$ until $21.5 \%$ where Hebert et al. (2003) have set a 10-fold sequence difference between the average interspecific and average intraspecific as a threshold for animal species identification.

Discrepancies in species assignment : A fraction of small dubious species assignments was detected on $7 \%$ of the specimen whereby they were assigned up to genus level only ( $<90 \%$ match) (Table 5). Afew studies have also found ambiguities and mismatches through DNA barcode data (Diaz et al., 2016; De Carvalho et al., 2011; Mabragaña et al., 2011). However, the 
Table 1 : Species assignment of 153 barcoded fish larvae from four mangrove areas surveyed in Peninsular Malaysia

\begin{tabular}{|c|c|c|c|c|c|c|c|}
\hline \multirow[t]{2}{*}{ Family } & \multirow[t]{2}{*}{ Species } & \multicolumn{2}{|l|}{$\%$} & \multicolumn{4}{|c|}{ Occurrence } \\
\hline & & $\begin{array}{l}\text { Genbank } \\
\text { Identity }\end{array}$ & $\begin{array}{l}\text { BOLD } \\
\text { Similarity }\end{array}$ & Pendas & Pekan & Matang & Setiu \\
\hline \multirow[t]{4}{*}{ Ambassidae } & Ambassis dussumieri & 95 & & & & & 1 \\
\hline & Ambassis marianus & 99 & & 4 & 5 & & \\
\hline & Ambassis nalua & 99 & & 1 & 1 & & \\
\hline & Ambassis sp. & 82 & 100 & & & & 1 \\
\hline Apogonidae & Ostorhinchus fasciatus & 99 & & 1 & & & \\
\hline \multirow[t]{3}{*}{ Clupeidae } & Hilsa kelee & 99 & & 2 & & & \\
\hline & Opisthonema libertate & 99 & & 2 & & & \\
\hline & Sardinella melanura & 100 & & 2 & & & \\
\hline Eleotridae & Ophiocara porocephala & - & 85 & & & 2 & \\
\hline \multirow[t]{3}{*}{ Engraulidae } & Thryssa kammalensis & 99 & & & 13 & & \\
\hline & Thryssa hamiltonii & 99 & & & 6 & & \\
\hline & Stolephorus indicus & 99 & & 1 & & 1 & \\
\hline Gerreidae & Gerres filamentosus & 93 & & 2 & & & \\
\hline \multirow[t]{14}{*}{ Gobiidae } & Boleophthalmus boddarti & 100 & & 2 & 1 & 2 & \\
\hline & Brachygobius kabiliensis & 100 & & 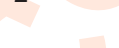 & & 1 & \\
\hline & Exyriaspuntang & 99 & & 1 & & & \\
\hline & Eugnathogobius variegatus & 99 & & & 1 & & \\
\hline & Hemigobius hoevenii & 99 & & & & 20 & \\
\hline & Glossogobius aureus & 99 & & 8 & & & \\
\hline & Gobiopterus lacustris & 99 & & & & 10 & \\
\hline & Mugilogobius chulae & 97 & & & 1 & & \\
\hline & Periophthalmus sp. & * & & & 1 & 1 & \\
\hline & Pseudogobiopsis oligactis & 97 & & & 2 & & \\
\hline & Pseudogobius javanicus & 99 & & 1 & 2 & & \\
\hline & Pseudogobius masago & 98 & & & 10 & 1 & \\
\hline & Pseudogobius olorum & 85 & & & & 10 & \\
\hline & Sicyopterussp. & 99 & & & 2 & 1 & \\
\hline \multirow[t]{2}{*}{ Leiognathidae } & Photopectoralis bindus & 99 & & 1 & & & \\
\hline & Leiognathus ruconius & 99 & & 1 & & & \\
\hline \multirow[t]{2}{*}{ Monacanthidae } & Monacanthus chinensis & 99 & & 1 & & & \\
\hline & Paramonacanthus sulcatus & 99 & & 1 & & & \\
\hline Mugilidae & Paramugil parmatus & 99 & & & 1 & & \\
\hline Mullidae & Upeneus margarethae & 99 & & & & & 1 \\
\hline Scatophagidae & Scatophagus argus & 100 & & 2 & 1 & & \\
\hline Sciaenidae & Dendrophysa russelii & 99 & & & & 1 & \\
\hline \multirow[t]{2}{*}{ Sillaginidae } & Sillago sihama & 99 & & & 1 & & \\
\hline & Sillago vittata & * & & 1 & & & \\
\hline Sparidae & Sparus aurata & 99 & & 15 & & & \\
\hline \multirow[t]{2}{*}{ Syngnathidae } & Hippichthys cyanospilos & 95 & & 1 & & & \\
\hline & Hippichthys penicillus & 94 & & 1 & & & \\
\hline \multirow[t]{2}{*}{ Tetraodontidae } & Chelonodon patoca & 98 & & 1 & & & \\
\hline & Undertermined & & & & & 3 & \\
\hline
\end{tabular}

availability of high-quality reference sequences in public sequence libraries (BOLD and GenBank) are important to avoid any discrepancies in confirmation of species. Lack of sufficient and adequate data will lead the blast searches to an ambiguous result. This situation might occur from misidentification of voucher specimen, contamination during sample processing, insufficient taxonomic identification or synonym and syntax problems (Ward, 2012; Raduloviciet al., 2010; Tautz et al., 2003).
In this study, three congeneric species of Pseudogobius; Pseudogobius masago, Pseudogobius olorum and Pseudogobius javanicus exhibited high average congeneric distance (mean distance $19.47 \%$, minimum distance $18.03 \%$ ), with two former species clustered as sister taxa, while Pseudogobius javanicus formed a separate branch in NJ (Fig. 2) and Bayesian (Fig. 4). In ML tree, all three species formed separate branch with unique set of $\mathrm{COI}$ haplotypes (Fig. 3). The 
Table 2: Summary of 98 observed mitochondrial DNA haplotypes in larval fish specimens from mangrove areas of Peninsular Malaysia, Malaysia

\begin{tabular}{|c|c|c|c|c|}
\hline Species & $\begin{array}{l}\text { BOLDSYSTEM index } \\
\text { Number (BIN) }\end{array}$ & $\begin{array}{l}\text { GenBank } \\
\text { accession }\end{array}$ & Sampling area & Sample ID \\
\hline Ambassis dussumieri & BOLD:ADB1883 & KX223884 & Setiu Terengganu & SA14A1 \\
\hline Ambassis marianus & BOLD:ACQ5401 & KX223888 & Pendas Johor & PR3A5_1 \\
\hline Ambassis marianus & BOLD:ACQ5401 & KX223889 & Pendas Johor & PC3A1 \\
\hline Ambassis marianus & BOLD:ACQ5401 & KX223887 & Pekan Pahang & CA1A1 \\
\hline Ambassis marianus & BOLD:ACQ5401 & KX223886 & Pendas Johor & PA1A4 \\
\hline Ambassis marianus & BOLD:ACQ5401 & KX223885 & Pekan Pahang & $\mathrm{CA} 2 \mathrm{~A} 2$ \\
\hline Ambassis marianus & BOLD:ACQ5401 & KX144849 & Pendas Johor & PP1A2 \\
\hline Ambassis nalua & BOLD:ADA4639 & KX223932 & Pendas Johor & PC2A6 \\
\hline Ambassis nalua & BOLD:ADA4639 & KX144850 & Pekan Pahang & САЗАЗ \\
\hline Ambassis sp. & BOLD:ACE0269 & KX223890 & Setiu Terengganu & Sp1A2 \\
\hline Boleophthalmus boddarti & BOLD:AAW7430 & KY849515 & Matang Perak & BBM2 \\
\hline Boleophthalmus boddarti & BOLD:AAW7431 & KY849544 & Matang Perak & BBM1 \\
\hline Boleophthalmus boddarti & BOLD:ADC7579 & KY849516 & Pekan Pahang & $\mathrm{BBC} 2$ \\
\hline Boleophthalmus boddarti & BOLD:AAW7430 & KY849511 & Pendas Johor & BBP1 \\
\hline Boleophthalmus boddarti & BOLD:AAW7431 & KY849537 & Pendas Johor & BBP8 \\
\hline Brachygobius kabiliensis & BOLD:ADB2718 & KX223927 & Matang Perak & MG92G12 \\
\hline Chelonodon patoca & BOLD:AAF2666 & KX223894 & Pendas Johor & PB1U1 \\
\hline Sillago vittata & BOLD:ADB2362 & KX223895 & Pendas Johor & PI1/1 \\
\hline Dendrophysa russelii & BOLD:AAD4938 & KX223896 & Matang Perak & Mp1Q1 \\
\hline Eugnathogobius variegatus & BOLD:AAW8350 & KX223930 & Pekan Pahang & CG36G19 \\
\hline Exyrias puntang & BOLD:ACT3463 & KX223899 & Pendas Johor & PG11G8 \\
\hline Gerres filamentosus & BOLD:AAM2324 & KX223900 & Pendas Johor & $\mathrm{PH} 1 \mathrm{H} 4$ \\
\hline Gerres filamentosus & BOLD:ADB1938 & KX281922 & Pendas Johor & PC4H1 \\
\hline Glossogobius aureus & BOLD:ADB2538 & KX223901 & Pendas Johor & PG1G4 \\
\hline Glossogobius aureus & BOLD:ADB2538 & KX281923 & Pendas Johor & PG23G11 \\
\hline Glossogobius aureus & BOLD:ADB2538 & KX223898 & Pendas Johor & PG22G10 \\
\hline Gobiopterus lacustris & BOLD:ADB1051 & KX223905 & Matang Perak & MG5G5 \\
\hline Gobiopterus lacustris & BOLD:ADB1051 & KX223902 & Matang Perak & MG4G4 \\
\hline Gobiopterus lacustris & BOLD:ADB1051 & KX281924 & Matang Perak & MG87G11 \\
\hline Gobiopterus lacustris & BOLD:ADB1051 & KX281925 & Matang Perak & MA3G32 \\
\hline Gobiopterus lacustris & BOLD:ADB1051 & KX223903 & Matang Perak & MG101G13 \\
\hline Hemigobius hoevenii & BOLD:ADD2192 & KX281927 & Matang Perak & MG120G33 \\
\hline Hemigobius hoevenii & BOLD:AAE5276 & KX223907 & Matang Perak & MA2G30 \\
\hline Hemigobius hoevenii & BOLD:AAE5276 & KX223906 & Matang Perak & MG13G35 \\
\hline Hemigobius hoevenii & BOLD:AAE5276 & KX281926 & Matang Perak & MG171G19 \\
\hline Hemigobius hoevenii & BOLD:AAE5276 & KX281928 & Matang Perak & MG160G18 \\
\hline Hemigobius hoevenii & BOLD:AAE5276 & KX223908 & Matang Perak & MG80G9 \\
\hline Hemigobius hoevenii & BOLD:AAE5276 & KX223909 & Matang Perak & MG64G38 \\
\hline Hilsa kelee & BOLD:AAC0856 & KX223912 & Pendas Johor & PE2K1 \\
\hline Hilsa kelee & BOLD:AAC0856 & KX223913 & Pendas Johor & PK4K3 \\
\hline Hippichthys cyanospilos & BOLD:ADB2646 & KX223915 & Pendas Johor & PW1Y1 \\
\hline Hippichthys penicillus & BOLD:ADB2645 & KX223914 & Pendas Johor & PW2Y2 \\
\hline Leiognathus ruconius & BOLD:ADB1713 & KX223916 & Pendas Johor & PS4L2 \\
\hline Monacanthus chinensis & BOLD:AAB6368 & KX223920 & Pendas Johor & PT1M1 \\
\hline Mugilogobius chulae & BOLD:ADB2817 & KX223921 & Pekan Pahang & CG18G3 \\
\hline Ophiocara porocephala & BOLD:ADB1822 & KX223922 & Matang Perak & MG54e2 \\
\hline Ophiocara porocephala & BOLD:ADB1822 & KX281930 & Matang Perak & MG47e1 \\
\hline Opisthonema libertate & BOLD:AAF2460 & KX223911 & Pendas Johor & PK1K2 \\
\hline Opisthonema libertate & BOLD:AAF2460 & KX223910 & Pendas Johor & PK12K6 \\
\hline Ostorhinchus fasciatus & BOLD:AAC1242 & KX223924 & Pendas Johor & PD1J \\
\hline Paramonacanthus sulcatus & BOLD:AAC5161 & KX223925 & Pendas Johor & PT2M2 \\
\hline Paramugil parmatus & BOLD:ABU7210 & KX223926 & Pekan Pahang & CN1m1 \\
\hline Periophthalmus sp. & BOLD:ADB2719 & KX223929 & Pekan Pahang & CG26G10 \\
\hline Periophthalmus sp. & BOLD:ADB2243 & KX223928 & Matang Perak & MG99G23 \\
\hline Photopectoralis bindus & BOLD:AAC5681 & KX223931 & Pendas Johor & PC1L1 \\
\hline
\end{tabular}


Pseudogobiopsis oligactis Pseudogobiopsis oligactis Pseudogobius javanicus Pseudogobius javanicus Pseudogobiusjavanicus Pseudogobius masago Pseudogobius masago Pseudogobius masago Pseudogobius masago Pseudogobius masago Pseudogobius masago Pseudogobius olorum Pseudogobius olorum Pseudogobius olorum Pseudogobius olorum Pseudogobius olorum Sicyopterus sp.

Sardinella melanura

Sardinella melanura

Scatophagus argus

Scatophagus argus

Scatophagus argus

Sicyopterussp.

Sicyopterus sp.

Sillago sihama

Sparus aurata

Sparus aurata

Sparus aurata

Sparus aurata

Sparus aurata

Stolephorus indicus

Stolephorus indicus

Thryssa hamiltonii

Thryssa hamiltonii

Thryssa hamiltonii

Thryssa hamiltonii

Thryssa hamiltonii

Thryssa hamiltonii

Thryssa kammalensis

Thryssa kammalensis

Thryssa kammalensis

Thryssa kammalensis

Upeneus margarethae

$\begin{array}{ll}\text { BOLD:ADB1314 } & \text { KX281931 } \\ \text { BOLD:ADB1314 } & \text { KX223933 } \\ \text { BOLD:AAD9462 } & \text { KX223936 } \\ \text { BOLD:AAD9462 } & \text { KX223937 } \\ \text { BOLD:AAD9462 } & \text { KX223935 } \\ \text { BOLD:ACC0583 } & \text { KX144852 } \\ \text { BOLD:ACC0583 } & \text { KX144851 } \\ \text { BOLD:ACC0583 } & \text { KX223938 } \\ \text { BOLD:ACC0583 } & \text { KX223939 } \\ \text { BOLD:ACC0583 } & \text { KX223940 } \\ \text { BOLD:ACC0583 } & \text { KX223941 } \\ \text { BOLD:ADB2106 } & \text { KX223943 } \\ \text { BOLD:ADB2106 } & \text { KX223944 } \\ \text { BOLD:ADB2106 } & \text { KX281936 } \\ \text { BOLD:ADB2106 } & \text { KX281934 } \\ \text { BOLD:ADB2106 } & \text { KX223942 } \\ \text { BOLD:AAE7961 } & \text { KX223934 } \\ \text { BOLD:AAB7198 } & \text { KX223945 } \\ \text { BOLD:ADB1709 } & \text { KX281937 } \\ \text { BOLD:AAB3531 } & \text { KX223948 } \\ \text { BOLD:AAB3531 } & \text { KX223947 } \\ \text { BOLD:AAB3531 } & \text { KX223946 } \\ \text { BOLD:ADB1338 } & \text { KX281932 } \\ \text { BOLD:ADB1338 } & \text { KX223893 } \\ \text { BOLD:ABZ1631 } & \text { KX223949 } \\ \text { BOLD:ADB2220 } & \text { KX223953 } \\ \text { BOLD:ADB2220 } & \text { KX223952 } \\ \text { BOLD:ADB2220 } & \text { KX223951 } \\ \text { BOLD:ADB2220 } & \text { KX223950 } \\ \text { BOLD:ADB2220 } & \text { KX223954 } \\ \text { BOLD:ADB1383 } & \text { KX281939 } \\ \text { BOLD:AAB7978 } & \text { KX223955 } \\ \text { BOLD:AAB6419 } & \text { KX223956 } \\ \text { BOLD:AAB6419 } & \text { KX281941 } \\ \text { BOLD:AAB6419 } & \text { KX281942 } \\ \text { BOLD:AAB6419 } & \text { KX281940 } \\ \text { BOLD:AAB6419 } & \text { KX223957 } \\ \text { BOLD:AAB6419 } & \text { KX223958 } \\ \text { BOLD:ADB1427 } & \text { KX223962 } \\ \text { BOLD:ADB1427 } & \\ \text { BOLD:ADB1427 } & \text { KOLD:ADB1427 } \\ \text { BOLD:AAB9714 } & \end{array}$

$\begin{array}{ll}\text { Pekan Pahang } & \text { CG17G2 } \\ \text { Pekan Pahang } & \text { CG35G16 } \\ \text { Pendas Johor } & \text { PG2G5 } \\ \text { Pekan Pahang } & \text { CG32G17 } \\ \text { Pekan Pahang } & \text { CG41G14 } \\ \text { Pekan Pahang } & \text { CG19G4 } \\ \text { Pekan Pahang } & \text { CG33G13 } \\ \text { Matang Perak } & \text { MG118G16 } \\ \text { Pekan Pahang } & \text { CG21G6 } \\ \text { Pekan Pahang } & \text { CG24G9 } \\ \text { Pekan Pahang } & \text { CG27G11 } \\ \text { Matang Perak } & \text { MG159G17 } \\ \text { Matang Perak } & \text { MG15G36 } \\ \text { Matang Perak } & \text { MG2G2 } \\ \text { Matang Perak } & \text { MG12G34 } \\ \text { Matang Perak } & \text { MG3G3 } \\ \text { Matang Perak } & \text { MA1G31 } \\ \text { Pendas Johor } & \text { PK6K4 } \\ \text { Pendas Johor } & \text { PK7K5 } \\ \text { Pendas Johor } & \text { PS1S4 } \\ \text { Pekan Pahang } & \text { CS1S1 } \\ \text { Pendas Johor } & \text { PS3S3 } \\ \text { Pekan Pahang } & \text { CG34G18 } \\ \text { Pekan Pahang } & \text { CG23G8 } \\ \text { Pekan Pahang } & \text { CG14Z1 } \\ \text { Pendas Johor } & \text { P13V1 } \\ \text { Pendas Johor } & \text { PI5V2_1 } \\ \text { Pendas Johor } & \text { PI7V4 } \\ \text { Pendas Johor } & \text { PI6V3 } \\ \text { Pendas Johor } & \text { PG9V7_1 } \\ \text { Matang Perak } & \text { MK15E1 } \\ \text { Pendas Johor } & \text { PK15E1 } \\ \text { Pekan Pahang } & \text { CE11E16 } \\ \text { Pekan Pahang } & \text { CE12E17 } \\ \text { Pekan Pahang } & \text { CE3E9 } \\ \text { Pekan Pahang } & \text { CE7E13 } \\ \text { Pekan Pahang } & \text { CE1E7 } \\ \text { Pekan Pahang } & \text { CE18E20 } \\ \text { Pekan Pahang } & \text { CG5E2 } \\ \text { Pekan Pahang } & \text { CG6E3 } \\ \text { Pekan Pahang } & \text { CG4E1 } \\ \text { Pekan Pahang } & \text { CG7E4 } \\ \text { SetiuTerengganu } & \text { SR1N1 } \\ & \end{array}$

Table 3 : Genetic divergences of Kimura 2-parameters (K2P) at different taxonomic level for fish larvae specimens from mangrove areas of Peninsular Malaysia, Malaysia

\begin{tabular}{lllllll}
\hline Comparison within & & \multicolumn{4}{c}{ Distance } \\
\cline { 5 - 7 } & Taxa & Comparisons & Min (\%) & Mean (\%) & $\operatorname{Max}(\%)$ & SE (\%) \\
\hline Species & 22 & 138 & 0 & 1 & 10.29 & 0.01 \\
Genus & 5 & 118 & 13.29 & 20.19 & 25.85 & 0.02 \\
Family & 5 & 924 & 16.01 & 23.31 & 30.96 & 0 \\
\hline
\end{tabular}

discordance formed might signal the presence of cryptic species among studied samples.

In the case of Sillago vittata from GenBank and larva of
Sillago vittata, each of the two nominal species, formed cohesive units in $\mathrm{NJ}$ and $\mathrm{ML}$ trees but were differently clustered in the Bayesian tree (Fig. 4) with genetic divergence value of $5.52 \%$, indicating the samples could be considered as two different 
Table 4 : DNA barcode gap between fish larvae in selected mangrove areas of Peninsular Malaysia, Malaysia

\begin{tabular}{|c|c|c|c|c|}
\hline Species & Mean intra-sp & Max intra-sp & Nearest species & Distance to NN \\
\hline Dendrophysa russelii & $N / A$ & $N / A$ & Ostorhinchus fasciatus & 26.01 \\
\hline Hilsa kelee & 0.49 & 0.49 & Opisthonema libertate & 19.5 \\
\hline Opisthonema libertate & 2.63 & 2.63 & Hilsa kelee & 19.5 \\
\hline Sardinella melanura & 2.79 & 2.79 & Hilsa kelee & 19.54 \\
\hline Stolephorus indicus & 2.79 & 2.79 & Hilsa kelee & 20.86 \\
\hline Thryssa hamiltonii & 1.46 & 3.46 & Thryssa kammalensis & 20.3 \\
\hline Thryssa kammalensis & 0.27 & 0.49 & Thryssa hamiltonii & 20.3 \\
\hline Ambassis dussumieri & $\mathrm{N} / \mathrm{A}$ & $\mathrm{N} / \mathrm{A}$ & Ambassis marianus & 13.29 \\
\hline Ambassis marianus & 0.49 & 0.97 & Ambassis dussumieri & 13.29 \\
\hline Ambassis nalua & 0 & 0 & Ambassis sp. & 21.76 \\
\hline Ambassis sp. & $\mathrm{N} / \mathrm{A}$ & $\mathrm{N} / \mathrm{A}$ & Ambassis nalua & 21.76 \\
\hline Ophiocara porocephala & 3.14 & 3.14 & Monacanthus chinensis & 20.09 \\
\hline Boleophthalmus boddarti & 0.89 & 1.92 & Sicyopterussp. & 20.13 \\
\hline Brachygobius kabiliensis & $\mathrm{N} / \mathrm{A}$ & $\mathrm{N} / \mathrm{A}$ & Monacanthus chinensis & 19.75 \\
\hline Eugnathogobius variegatus & $\mathrm{N} / \mathrm{A}$ & N/A & Pseudogobius olorum & 19.14 \\
\hline Exyrias puntang & $\mathrm{N} / \mathrm{A}$ & $\mathrm{N} / \mathrm{A}$ & Scatophagus argus & 20.52 \\
\hline Glossogobius aureus & 0.43 & 0.65 & Exyrias puntang & 20.9 \\
\hline Gobiopterus lacustris & 0.49 & 0.81 & Sicyopterussp. & 20.21 \\
\hline Hemigobius hoevenii & 1.09 & 2.62 & Pseudogobius olorum & 18.95 \\
\hline Mugilogobius chulae & $\mathrm{N} / \mathrm{A}$ & $\mathrm{N} / \mathrm{A}$ & Sicyopterus sp. & 16.01 \\
\hline Periophthalmus sp. & 4.51 & 4.51 & Mugilogobius chulae & 19.85 \\
\hline Pseudogobiopsis oligactis & 1.14 & 1.14 & Sillago sp. & 20.93 \\
\hline Pseudogobiusjavanicus & 0.43 & 0.65 & Mugilogobius chulae & 16.9 \\
\hline Pseudogobius masago & 0.4 & 0.65 & Pseudogobius olorum & 18.03 \\
\hline Pseudogobius olorum & 1.07 & 1.63 & Sicyopterus sp. & 17.5 \\
\hline Sicyopterussp. & 6.8 & 10.29 & Mugilogobius chulae & 16.01 \\
\hline Ostorhinchus fasciatus & $N / A$ & $N / A$ & Ambassis marianus & 19.58 \\
\hline Paramugil parmatus & $\mathrm{N} / \mathrm{A}$ & $N / A$ & Monacanthus chinensis & 19.61 \\
\hline Gerres filamentosus & 2.79 & 2.79 & Ambassis dussumieri & 21.5 \\
\hline Leiognathus ruconius & $\mathrm{N} / \mathrm{A}$ & $N / A$ & Photopectoralis bindus & 22.07 \\
\hline Photopectoralis bindus & $\mathrm{N} / \mathrm{A}$ & $N / A$ & Sillago sp. & 21.77 \\
\hline Upeneus margarethae & $\mathrm{N} / \mathrm{A}$ & $\mathrm{N} / \mathrm{A}$ & Monacanthus chinensis & 20.52 \\
\hline Scatophagus argus & 0.32 & 0.48 & Ambassis marianus & 19.29 \\
\hline Sillago sihama & $\mathrm{N} / \mathrm{A}$ & $\mathrm{N} / \mathrm{A}$ & Hilsa kelee & 20.4 \\
\hline Sillago sp. & $\mathrm{N} / \mathrm{A}$ & $N / A$ & Pseudogobiopsis oligactis & 20.93 \\
\hline Sparus aurata & 0.68 & 1.47 & Sillagosp. & 21.99 \\
\hline Hippichthys cyanospilos & $\mathrm{N} / \mathrm{A}$ & $\mathrm{N} / \mathrm{A}$ & Hippichthys penicillus & 19.09 \\
\hline Hippichthys penicillus & $\mathrm{N} / \mathrm{A}$ & $N / A$ & Hippichthys cyanospilos & 19.09 \\
\hline Monacanthus chinensis & $\mathrm{N} / \mathrm{A}$ & $\mathrm{N} / \mathrm{A}$ & Paramonacanthus sulcatus & 16.24 \\
\hline Paramonacanthus sulcatus & $\mathrm{N} / \mathrm{A}$ & $N / A$ & Monacanthus chinensis & 16.24 \\
\hline Chelonodon patoca & $\mathrm{N} / \mathrm{A}$ & $\mathrm{N} / \mathrm{A}$ & Paramugil parmatus & 22.99 \\
\hline
\end{tabular}

Note: N/Ais not available.

Table 5 : Nearest match in identification of fish larvae specimens from mangrove areas of Peninsular Malaysia, Malaysia

\begin{tabular}{llllll}
\hline Query Species & \multicolumn{2}{c}{ GenBank } & & \multicolumn{2}{c}{ BOLD } \\
\cline { 2 - 3 } & Species assigment & Identity (\%) & & Species assigment & Similarity (\%) \\
\hline Ambassis sp. & Ambassis marianus & 82 & & Ambassis nalua & 85 \\
Sillago vittata & Cirripectes stigmatus & 84 & & Sillago sp. & Unresolved \\
Periophthalmus sp. & Periophthalmus modestus & 84 & & Periophthalmus kalolo & 82 \\
Ophiocara porocephala & Lutjanus sp. & 83 & & Ophiocara porocephala & 85 \\
Pseudogobius olorum & Pseudogobius olorum & 85 & & Pervagoraspricaudus & 85 \\
\hline
\end{tabular}




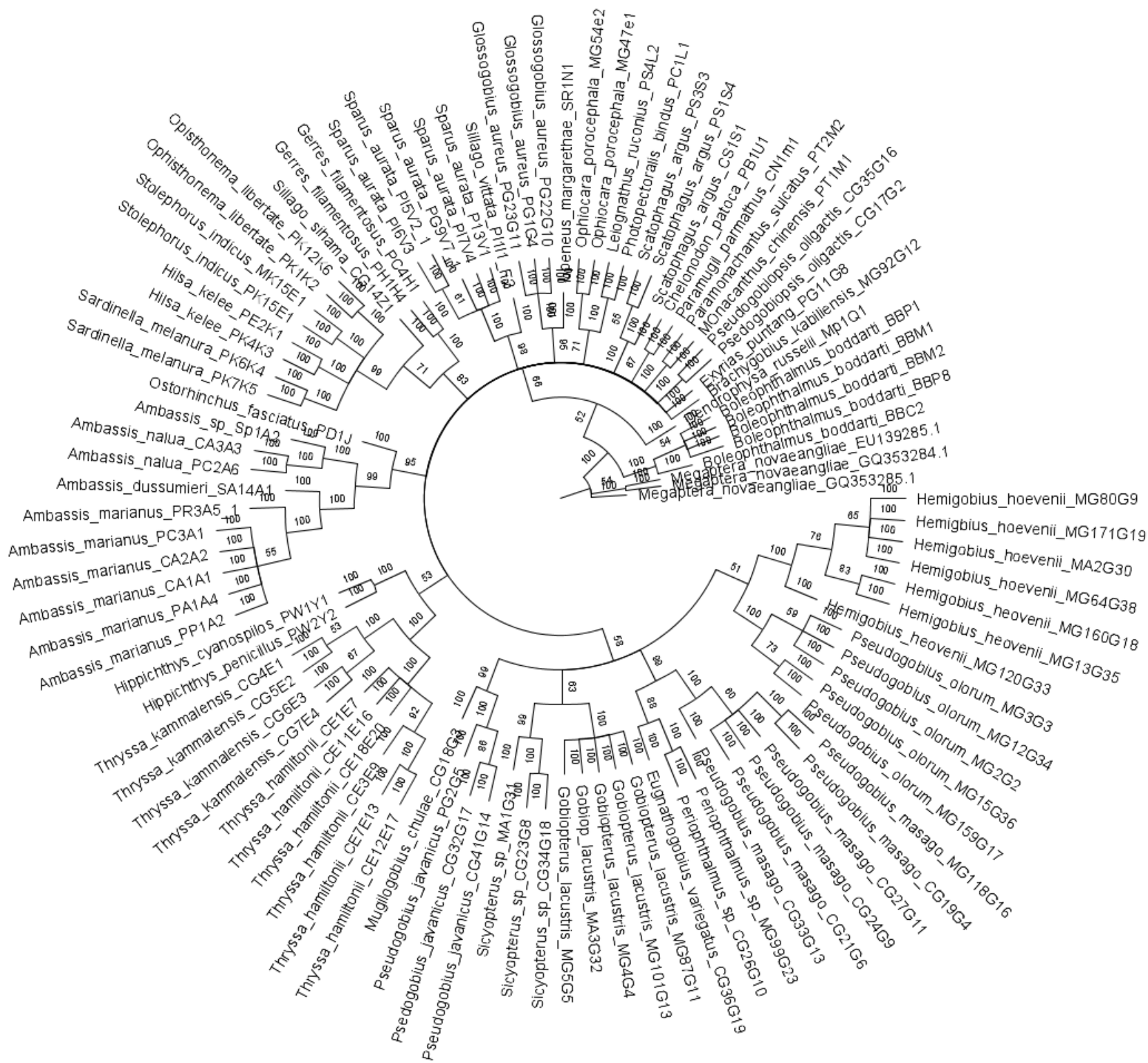

Fig. 2 : Neighbour-Joining (NJ) tree showing relationships among 98 haplotypes of fish larvae sampled from selected mangrove areas in Peninsular Malaysia. Each node represented with bootstrap value (\%) after 1000 pseudoreplications of $\mathrm{NJ}$ analysis.

species. A similar case of deep genetic divergence between the two species also occurred between the Brazilian cusk eel, Genypterus brasiliensis and the Pink cusk-eel, G. blacodes. The finding was further supported by both morphological and molecular approaches proving that Genypterus blacodes and G. brasiliensis should be pronounced as a separate species (Mabragaña et al., 2011). Hebert et al., (2003) demonstrated that collection of multiple samples within each species would help to avoid paraphyly or polyphyly assignment of a species, as in case of sillago vittata. Simultaneously, the lack of large and robust database with reliable reference sequences might be the reason for this shortcoming similar to the situation faced in the study of
Indo-Pacific larvae by Leis (2015) and fish larvae study in Eastern Atlantic Ocean by Ardura et al. (2016).

Vague species assignment also occurred in few specimens where the issue stems from recent taxonomic changes in species nomenclature, for example in the cases of Paramugil parmatus/Liza melinoptera and Pseudogobius oligactis/Eugnathogobius oligactis. Both cases were found to be a synonym name of assigned species whereby the former assigned name was most updated naming used (Fishbase.org, 2017). According to Pusey (2001), synonymy simply occurrs when naming of the species is done in different manner by two or 


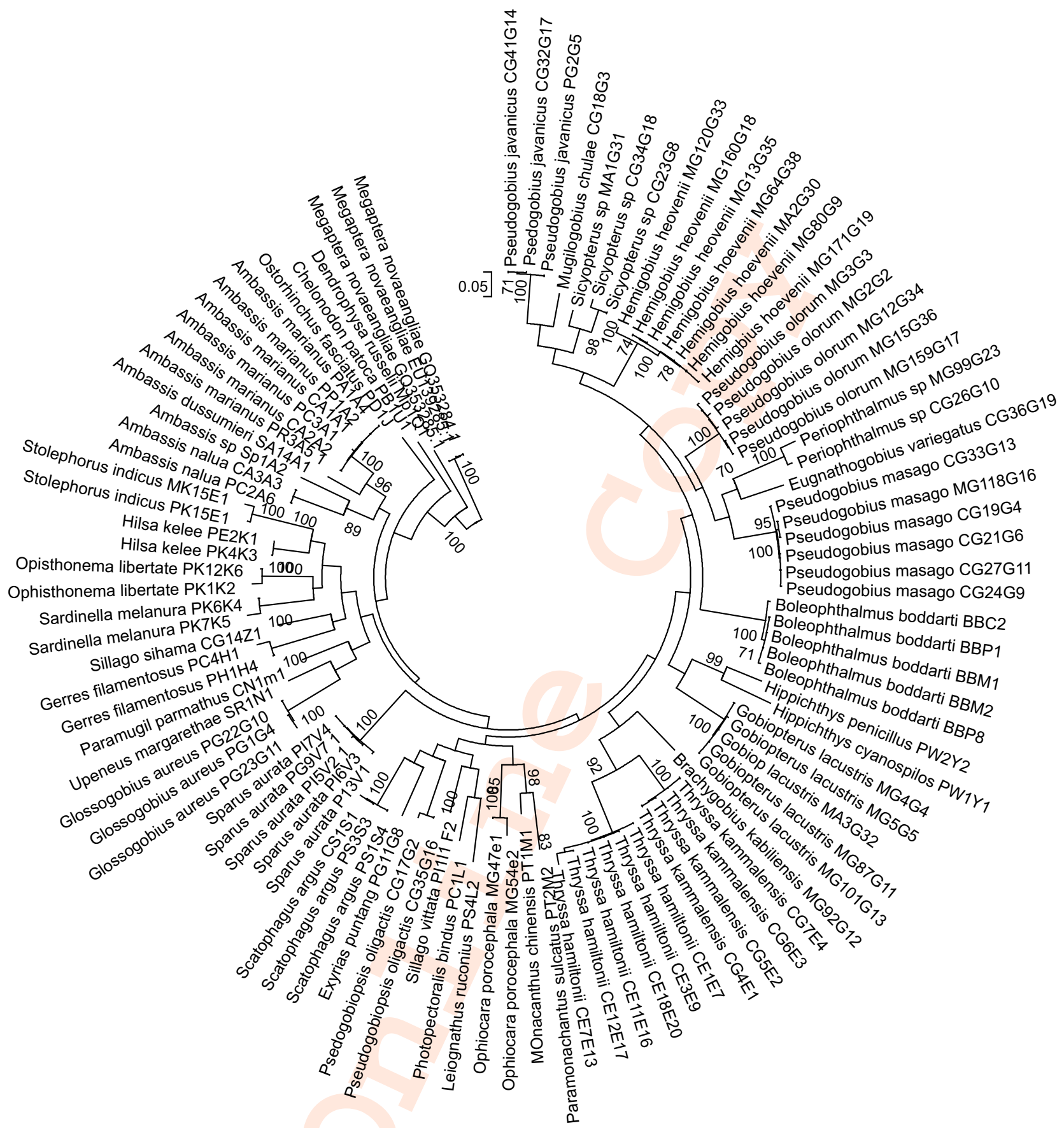

Fig. 3 : Maximum likelihood (ML) tree showing relationships among 98 haplotypes of fish larvae sampled from selected mangrove areas in Peninsular Malaysia. Each node represented with bootstrap value (\%) after 1000 of pseudoreplications.

more authors. This situation will require revision to make sure that the nomenclature remains correct, relevant and accurately reflects evolutionary history.

High values of intraspecific genetic divergence $(>2 \%)$ : There were several species displaying $>2 \%$ intraspecific divergence where 9 species $(21.95 \%)$ were further subdivided into two or more sublusters. Each cluster showed a strong bootstrap value of
100\%, except in Thryssa hamiltonii and Hemigobius hoevenii. Deep intraspecific divergence has also been reported in few other barcoding studies involving bird (Milá et al., 2012), scorpion (Coelho et al., 2014), mollusk (Ye et al., 2015) and cichlid fish (Colatreli et al., 2012) which suggested underestimation of species diversity.

Sardinella melanura (2.79\%), Stolephorus indicus 


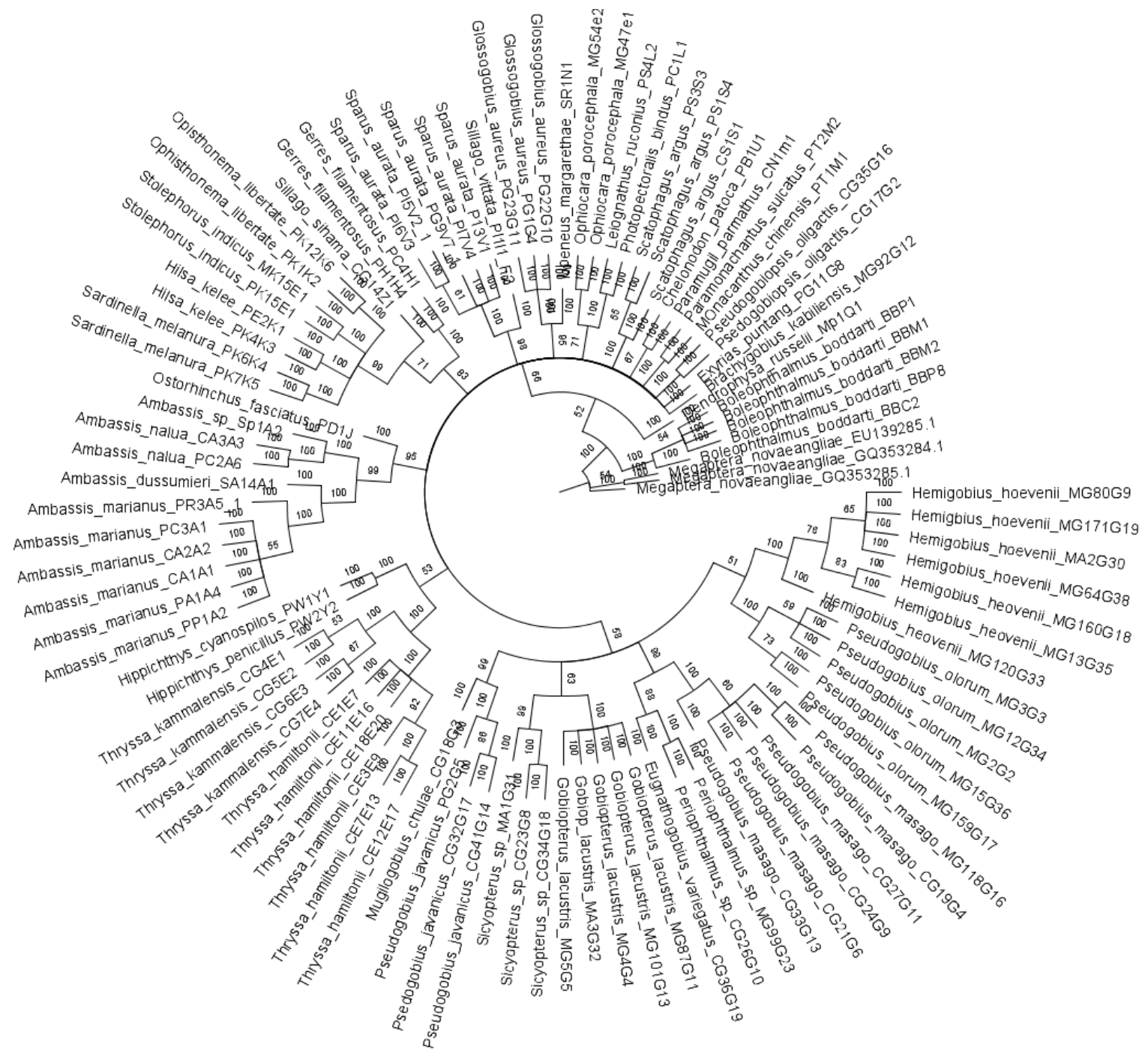

Fig. 4 : Bayesian inference (BI) showing relationships among 98 haplotypes of fish larvae sampled from selected mangrove areas in Peninsular Malaysia.

$(2.79 \%)$ and Opisthonema libertate $(2.63 \%)$ belonged to the Clupeiformes order with a worldwide distribution of 402 described species in Indo-West Pacific Ocean inhabiting brackish and marine waters and also freshwater (Li and Orti, 2007). However, in the study of Hebert et al. (2003), species divergence cut-off value of $3 \%$ was applied in the lepidoteran species delimitation resulting in $98 \%$ success on species recognition. Due to this, Gerres filamentosus $(2.79 \%)$ and the rest of species with below $3 \%$ intraspecific genetic divergence value were still considered as morphospecies, thus their species assignment was not to be contested.

The status of Thryssa hamiltonii (mean, 1.46\%, max $3.46 \%$ ) species assignment was in question from biogeographical perspective, as whether they were sampled from local populations or they were transported from the oceanic currents. T. hamiltonii was believed to be transported from distant spawning locations as Clupeiformes larvae have a long planktonic life of 6 weeks to 8 weeks (Smith et al., 1977) and members of this family have high plasticity in its habitat use for reproduction making them a facultative diadromous fish (Mai and Vieira, 2013).

Four species reported in this study represented mangrove resident fishes, the Ophiocara porocephala (mean $3.14 \%$ ) from Eleotridae family while Hemigobius hoevenii (mean 1.19\%, max 2.62\%), Periophthalmus sp. (mean 4.51\%) and Sicyopterus sp. (mean 6.8\%, max 10.29\%) were gobiids. The gobiids are highly diverse family and especially rich in the Indo-Pacific region, including freshwaters of the Indo-Malay Archipelago (Larson et al., 2014). The gobiids have faced a lot of 
morphological ambiguities as compared to other fish families for example Terapontidae, Sillaginidae and Ambassidae (Krishna et al., 2012). Sicyopterus pugnans and Sicyopterus lagocephalus has been reported in neighbouring region, the Philipines (Bayot et al., 2014), hence shed a light of possible species assignment for Sicyopterus sp. in this study. Genetic divergence between three specimens of Sicyopterus sp. was found in a huge gap as they were collected from different region of Peninsular Malaysia (2 samples from East Malaysia and one from West Malaysia).

In some species sharp genetic discontinuities between two regions may occur in the absence of dispersal and gene flow, such as discontinuities in the oceanographic regimes (Aziz et al., 2016, Gonzalez et al., 2016) which later influence their genetic divergence and population structure (Li et al., 2017). As for Periophthalmus sp., strong bootstrap value in $\mathrm{ML}$ and $\mathrm{NJ}$ supported the monophyletic relationship between the two specimens, however, due to insufficient records of DNA barcode reference libraries in BOLD for Indo-Pacific fishes, the broad use of libraries in automated identification became limited (Hubert et al., 2012).

Since barcodes of same sequences of species invariably were clustered in the same clade, despite the differences in locality (one specimen from the West Peninsular Malaysia and the other from East Peninsular Malaysia), Krishna et al. (2012) concluded that across geography barcodes of same species do not contain much variations. However, high conspecific divergences occasionally may reflect actual cases of undescribed species or sub-species (Landi et al., 2014). For example, members of gobiids from Oxudercinae subfamilies was claimed as not a well-known taxa (Okiyama, 1988) were considered to have ambiguity in their morphological taxonomy (Krishna et al., 2012) in which Leis and Carson-Ewart (2000) claimed to have lacked in reference materials resulted in no description was able to be drawn for the group.

Thus, from this study it can be concluded that molecular methods in particular DNA barcoding is the best method for the identification of fish larvae.

Even though, previously molecular method was considered intimidating to fellow taxonomists, integration of both methods is expected to do wonders in solving the identity of phenotypically ambiguous sample. The integrative method should receive good support from future researchers as it was not undermining any field of expertise and in fact will help solve more unsolved cases, especially for fish larvae.

\section{Acknowledgments}

This paper was a part of doctoral thesis of Izzati Adilah Azmir. The first author would like to thank South east Asian
Regional Center for Graduate Study and Research in Agriculture (SEARCA) for the scholarship award for conducting the project. This project was funded by Fundamental Research Grant Scheme (FRGS) 07-02-141544FR and LRGS/1/2019/UPM/1 (vote no. 5545102) funded by the Ministry of Education, Malaysia.

\section{References}

Agorreta, A., D.S. Mauro, U. Schliewen, J.L.V. Tassell, M. Kovačić, R. Zardoya and L. Rüber: Molecular phylogenetics of Gobioidei and phylogenetic placement of European gobies. Molec. Phylog. Evol., 69, 619-633 (2013).

Ajmal Ali, M., G. Gyulai, N. Hidvegi, B. Kerti, F.M. Al Hemaid, A.K. Pandey and J. Lee: The changing epitome of species identification-DNA barcoding. Saudi J. Biol. Sci., 21, 204-231 (2014).

Ara. R., A. Arshad, S.M.N. Amin, S.K. Daud and M.A. Ghaffar: Environment and diversity of ichthyoplankton in the seagrass beds of Sungai Pulai estuary, Johor Peninsular Malaysia. J. Food, Agric. Environ., 9, 733-738 (2011).

Ardura, A., E. Morote, M. Kochzius and E.G. Vazquez: Diversity of planktonic fish larvae along a latitudinal gradient in the Eastern Atlantic Ocean estimated through DNA barcodes. PeerJ., 4, e2438 (2016).

Atan, Y., H. Jaafar and A.R.A. Majid: Ikan Laut Malaysia glosari nama sahih spesies ikan. Dewan Bahasa dan Pustaka (2010).

Avise, J.C.: Molecular Markers, Natural History, and Evolution. Chapman \& Hall, New York, pp. 511(1999).

Aziz, N.M.A., Y. Esa and A. Arshad: DNA barcoding and phylogenetic analysis of Malaysian groupers (Subfamily: Epinephelinae) using mitochondrial Cytochrome c oxidase I (COI) gene. J. Environ. Biol., 37, 725-733 (2016).

Azmir, I., Y. Esa, S.M.N. Amin, I.S. Md Yasin and F. Md Yusof: Identification of larval fish in mangrove areas of Peninsular Malaysia using morphology and DNA barcoding methods. J. Appl. Ichthyol., 33, 998-1006 (2017).

Bayot, F.A., K.N.A. Joya and W.R. Jacinto: Phylogenetic analysis and barcoding of gobies in Maragondon river, Cavite, Philippines. Inter. J. Curr. Sci., 12, 50-6 (2014).

Branicki, W., Kupiec, T. and Pawlowski, R.: Validation of cytochrome b sequence analysis as a method of species identification. $J$. Forens. Sci., 48, 1-5 (2003).

Chu, C., K.H. Loh, C.C. Ng, A.L. Ooi, Y. Konishi, S.P. Huang and V.C. Chong: Using DNA Barcodes to Aid the Identification of Larval Fishes in Tropical Estuarine Waters (Malacca Straits, Malaysia). Zool. Stud., 58, e30 (2019).

Coelho, P., P. Sousa, D.J. Harris and A.V. der Meijden: Deep intraspecific divergences in the medically relevant fat-tailed scorpions (Androctonus, Scorpiones). Acta Tropica., 134, $43-51$ (2014).

Colatreli, O.P., N.V. Meliciano, D. Toffoli, I.P. Farias and T. Hrbek: Deep phylogenetic divergence and lack of taxonomic concordance in species of Astronotus (Cichlidae). Int. J. Evol. Biol., 915265 (2012).

De Carvalho, D.C., D.A. Oliveira, P.S. Pompeu, C.G. Leal, C. Oliveira and R. Hanner: Deep barcode divergence in Brazilian freshwater fishes: the case of the São Francisco River basin. Mitoch. DNA, 22, 80-86 (2011).

Díaz, J., G.V. Villanova, F. Brancolini, F. del Pazo, V.M. Posner, A. Grimberg and S.E. Arranz: First DNA barcode reference library for 
the identification of South American freshwater fish from the lower Paraná river. PloS ONE, 11, e0157419 (2016).

Fishbase. Boleophthalmus boddarti. Accessed on $26^{\text {th }}$ May 2017. http://www.fishbase.org/summary/Boleophthalmus-boddarti.html

Foon, J.K., S.K. Tan and G.R. Clements: A new species of Kenyirus (Pulmonata, Camaenidae) from Peninsular Malaysia. J. Trop. Biol. Conser., 12, 25-34 (2015)

Freitas, D.M. and J.H. Muelbert: Ichthyoplankton distribution and abundance off Southeastern and Southern Brazil. Brazil. Archi. Biol. Technol., 47, 601-612 (2004).

Gonzalez, E.B., H. Knutsen and P.E. Jorde: Habitat discontinuities separate genetically divergent populations of a rocky shore marine fish. PloS ONE, 11, e0163052 (2016).

Hall, T. A.: BioEdit: A user-friendly biological sequence alignment editor and analysis program for Windows 95/98/NT. In: Nucleic Acids Symposium Series, London. Information Retrieval Ltd., 41, pp. 9598 (1999).

Hebert, P.D., A. Cywinska, S.L. Ball and J.R. DeWaard: Biological identifications through DNA barcodes. Proc. R. Soc. Lond. B, 270, 313-21 (2003)

Hebert, P.D., E.H. Penton, J.M. Burns, D.H. Janzen and W. Hallwachs: Ten species in one: DNA barcoding reveals cryptic species in the neotropical skipper butterfly Astraptes fulgerator. Proceedings of the National Academy of Sciences of the United States of America., 101, 14812-14817 (2004a).

Hebert, P.D., M.Y. Stoeckle, T.S. Zemlak and C.M. Francis: Identification of birds through DNA barcodes. PLoS Biol., 2, e312 (2004b).

Hubert, N., C.P. Meyer, H.J. Bruggemann, F. Guérin, R.J.L. Komeno, B. Espiau, R. Causse, J.T. Williams and S. Planes: Cryptic diversity in Indo Pacific coral-reef fishes revealed by DNA-barcoding provides new support to the centre-of-overlap hypothesis. PLOS ONE, 7, e28987 (2012)

Huelsenbeck, J.P. and F. Ronquist: MRBAYES: Bayesian inference of phylogenetic trees. Bioinformatics, 17, 754-755 (2001).

Kimura, M.: A simple method for estimating evolutionary rates of base substitutions through comparative studies of nucleotide sequences. J. Mole. Evol., 16, 111-120 (1980).

Krishna, P.V., K.M. Rao and D.S. Rao: Identification of selected estuarine fishes by DNA barcoding from river Krishna region, Andhra Pradesh, India. Inter. J. Res. Pharmace. Biomed. Sci., 3, 10441049 (2012)

Landi, M., M. Dimech, M. Arculeo, G. Biondo, R. Martins, M. Carneiro, G.R. Carvalho, S.L. Brutto and F.O. Costa: DNA barcoding for species assignment: The case of Mediterranean marine fishes. PLoS One., 9, e106135 (2014).

Larkin, M. A., G. Blackshields, N.P. Brown, R. Chenna, P.A. McGettigan, H. McWilliam, F. Valentin, I.M. Wallace, A. Wilm, R. Lopez and J.D. Thompson: Clustal $\mathrm{W}$ and Clustal $\mathrm{X}$ version 2.0. Bioinformatics, 23, 2947-2948 (2007).

Larson, G., D.R. Piperno, R.G. Allaby, M.D. Purugganan, L. Andersson, M.A. Kalin, L. Barton, C.C. Vigueira, T. Denham, K. Dobney, A.N. Doust, P. Gepts, M.T. Gilbert, K.J. Gremillion, L. Lucas, L. Lukens, F.B. Marshall, K.M. Olsen, J.C. Pires, P.J. Richerson, R.R. de Casas, O.I. Sanjur, M.G. Thomas and D.Q. Fuller: Current perspectives and the future of domestication studies. Proc. Natl. Acad. Sci., 111, 6139-46 (2014).

Leis, J.M. and B.M.C. Ewart: The larvae of Indo-Pacific Coastal Fishes: An identification guide to marine fish larvae. Brill Publisher, Leiden, The Netherlands. p. 850 (2000).
Leis, J. M., and McCormic, M. I.: The biology, behaviour and ecology of the pelagic, larval stage of coral reef fishes. In: Coral Reef Fishes. (Ed.: P.J. Sale). Elsevier Science, USA. pp. 171-199 (2002).

Leis, J.M.: Taxonomy and systematics of larval Indo-Pacific fishes: a review of progress since 1981. Ichthyol. Res., 62, 9-28 (2015).

Lewis, L. A., Richardson, D. E., Zakharov, E. V. and Hanner, R.: Integrating DNA barcoding of fish eggs into ichthyoplankton monitoring programs. Fishery Bulletin, 114, 153-166 (2016).

Li, C. and G. Ortí: Molecular phylogeny of Clupeiformes (Actinopterygii) inferred from nuclear and mitochondrial DNA sequences. Molec. Phyloge. Evolut., 44, 386-398 (2007).

Li, Y., A. Ludwig and Z. Peng: Geographical differentiation of the Euchiloglanis fish complex (Teleostei: Siluriformes) in the Hengduan Mountain Region, China: Phylogeographic evidence of altered drainage patterns. Ecol. Evol., 7, 928-940 (2017).

Lima, A.R.A., M. Barletta, M.F. Costa, J.A.A. Ramos, D.V. Dantas, P.A.M.C. Melo, A.K.S. Justino and G.V.B. Ferreira: Changes in the composition of ichthyoplankton assemblage and plastic debris in mangrove creeks relative to moon phases. J. Fish Biol., 89, 619640 (2015).

Mabragaña, E., J.M.D. de Astarloa, R. Hanner, J. Zhang and M.G. Castro: DNA barcoding identifies Argentine fishes from marine and brackish waters. PLOS ONE, 6, e28655 (2011).

Mansor, M.I., H. Kohno, H. Ida, H.T. Nakamura, Z. Aznan and S. Abdullah: Field Guide to Important Commercial Marine Fishes of the South China Sea. Malaysia: Marine Fishery Resources Development and Management SEAFDEC., MFRDMD/SP/2. (1998).

Mat Jaafar, T.N., M.I. Taylor, S.A. Mohd Nor, M. de Bruyn and G.R. Carvalho: DNA barcoding reveals cryptic diversity within commercially exploited Indo-Malay Carangidae (Teleosteii: Perciformes). PLoS ONE, 7, e49623 (2012).

Mai, A.C.G. and J.P. Vieira: Review and consideration on habitat use, distribution and life history of Lycengraulis grossidens (Agassiz, 1829) (Actinopterygii, Clupeiformes, Engraulididae). Biota Neotro., 13, 121-130 (2013).

Milá, B., E.S. Tavares, A.M. Saldaña, J. Karubian, T.B. Smith and A.J. Baker: A trans-Amazonian screening of mtDNA reveals deep intraspecific divergence in forest birds and suggests a vast underestimation of species diversity. PLOS ONE., 7, e40541 (2012).

National Hydrographic Malaysia.: Tide Tables Malaysia 2015. Volume 1. Malaysia. (2015)

Okiyama, M.: An atlas of the early stage fishes in japan. Tokai University Press, Tokyo, Japan. p. 1154 (1988).

Pereira, L.H., R. Hanner, F. Foresti and C. Oliveira: Can DNA barcoding accurately discriminate megadiverse Neotropical freshwater fish fauna. BMC Genetics., 14, 20 (2013).

Pusey, B.: The new and the known: describing freshwater fish species. Using Rainforest Research., p. 2 (2001). Retrieved from http://www.wettropics.gov.au/site/user-assets/docs/new Fishes.pdf

Radulovici, A.E., P. Archambault and F. Dufresne: DNA barcodes for marine biodiversity: moving fast forward. Diversity, 2, 450-472 (2010).

Ratnasingham, S. and P.D.N. Hebert: Barcoding Bold: The barcode of life data system (www.barcodinglife.org). Mole. Ecol. Not., 7, 355-364 (2007).

Russell, F.S.: The eggs and planktonic stages of british marine fish. 
Academic Press, London, New York, San Francisco. p. 524 (1976).

Saitou, N. and M. Nei: The neighbor-joining method: A new method for reconstructing phylogenetic trees. Mole. Biol. Evol., 4, 406-425 (1987).

Sandilyan, S. and Kathiresan, K.: Decline of mangroves - A threat of heavy metal poisoning. Ocean \& Coastal Management, 102, 161168 (2014).

Shao, K.T., K.C. Chen and J.H. Wu: Identification of marine fish eggs in Taiwan using light microscopy, scanning electric microscopy and mtDNA sequencing. Mar. Fresh. Res., 53, 355-365 (2002).

Shen, Y., L. Guan, D. Wang and X. Gan: DNA barcoding and evaluation of genetic diversity in Cyprinidae fish in the midstream of the Yangtze River. Ecol. Evol., 6, 2702-2713 (2016).

Smith, R.L. and J.A. Cameron: Acute Effects-Pacific herring roe in the Gulf of Alaska. Environmental assessment of the alaskan continental shelf. Annual Reports, 12, 596-635 (1977).

Tan, K.H.: Protection and management of mangroves in Malaysia: Challenges and strategies. In: Proceedings of national conference on coastal and marine biodiversity, Kuala Lumpur (2007).

Tautz, D., P.Arctander, A. Minelli, R.H. Thomas and A.P. Vogler:A plea for DNA taxonomy. Tren. Ecol. Evolut., 18, 70-74 (2003).

Trivedi, S., A.A. Aloufi, A.A. Ansari and S.K. Ghosh: Role of DNA barcoding in marine biodiversity assessment and conservation: an update. Saudi J. Biol. Sci., 23, 161-171 (2016).

Valdez-Moreno, M., L. Va'squez-Yeomans, M. Elı'as-Gutie'rrez, N.V. Ivanova and P.D.N. Hebert: Using DNA barcodes to connect adults and early life stages of marine fishes from the Yucatan Peninsula, Mexico: potential in fisheries management. Mar. Freshwater Res., 61,665-671 (2010).

Ward, R.D., T.S. Zemlak, B.H. Innes, P.R. Last and P.D. Hebert: DNA barcoding Australia's fish species. Phil. Trans. R. Soc. B, 360, 1847-1857 (2005).

Ward, R.D., F.O. Costa, B.H. Holmes and D. Steinke: DNA barcoding of shared fish species from the North Atlantic and Australasia: minimal divergence for most taxa, but Zeus faber and Lepidopus caudatus each probably constitute two species. Aqua. Biol., 3, 7178 (2008).

Ward, R.D.: FISH-BOL, A case study for DNA barcodes. In: DNA Barcodes: Methods and Protocols, Methods in Molecular Biology (Eds: W.J. Kress and D.L. Erickson). Springer Science+Business Media, LLC, New York. 858, 423-439 (2012).

Wellington, G.M. and B. Victor: Planktonic larval duration of one hundred species of Pacific and Atlantic damsel fishes (Pomacentridae). Mar. Biology., 101, 557-567 (1989)

Winterbottom, R.: Search for the gobi old sister group (Actinopterygii: Percomorpha). Bull. Mar. Sci., 52, 395-414 (1993).

Xia, X.: DAMBE5: A comprehensive software package for data analysis in molecular biology and evolution. Molec. Biol. Evol., 30, 17201728 (2013).

Ye, Y.Y., C.W. Wu and J.J. Li: Genetic population structure of Macridiscus multifarius (Mollusca: Bivalvia) on the basis of mitochondrial markers: strong population structure in a species with a short planktonic larval stage. PloS ONE, 10, e0146260. 